\title{
Can the Timing of Ileostomy Reversal Influence Functional Outcome?
}

\author{
Chang-Nam Kim \\ Department of Surgery, Eulji University School of Medicine, Daejeon, Korea
}

See Article on Page 23-28

During rectal cancer surgery, we often make a temporary defunctioning stoma like an ileostomy or a colostomy to protect the anastomosis. Generally, as part of the primary surgery, an ileostomy is more common than a colostomy because the construction and reversal of an ileostomy is easier than that of a colostomy and because an ileostomy is associated with fewer stoma-related complications than a colostomy is. As soon as the primary surgery is finished, many patients frequently ask about the timing of an ileostomy reversal. They are eager to undergo an ileostomy reversal as early as possible because an ileostomy is a very stressful condition by itself to the patients. At present, no consensus exists for the optimal timing for an ileostomy reversal. Therefore, the timing of an ileostomy closure can be exceedingly inconsistent among hospitals. Chand et al. [1] reported on timely closure of an ileostomy for patients who had undergone rectal cancer surgeries. They compared time to closure of the ileostomy between two centers. Center 1 had a plan to provide patients with a temporary date for ileostomy reversal: (1) if there are no reasons for delay, ileostomy closure will be performed 6-8 weeks after discharge; (2) for patients who undergo adjuvant chemotherapy, ileostomy reversal will be scheduled between the second and the third cycles of treatment (still within 12 weeks). Center 2 had a traditional follow-up in the outpatient clinic. Of the total 82 patients in the study, 30 patients (37\%) underwent ileostomy closure within 12 weeks: In center 1, 23 of 34 patients (67\%) underwent ileostomy closure within 12 weeks, but in center 2, just 7 of 48 patients (15\%) underwent ileostomy reversal within 12 weeks. At 1 year, all patients in center 1 had under-

Correspondence to: Chang-Nam Kim, M.D.

Department of Surgery, Eulji University Hospital, Eulji University School of

Medicine, 95 Dunsan-seoro, Seo-gu, Daejeon 302-799, Korea

Tel: +82-42-259-1335, Fax: +82-42-259-1335

E-mail: kimcn@eulji.ac.kr

(C) 2015 The Korean Society of Coloproctology

This is an open-access article distributed under the terms of the Creative Commons Attribution NonCommercial License (http://creativecommons.org/licenses/by-nc/3.0) which permits unrestricted noncommercial use, distribution, and reproduction in any medium, provided the original work is properly cited. gone ileostomy closure, but in center 2, 5 of 48 patients (10\%) had not. The study concluded that the majority of ileostomies were closed within 12 weeks when the patients were provided with a date for ileostomy reversal at discharge after the primary rectal surgery.

Although many surgeons generally perform an ileostomy reversal around 3 months after the primary rectal surgery, for many reasons, the operation sometimes may be delayed by more than 3 months. den Dulk et al. [2] reported factors that limited stoma reversal in patients who had undergone a total mesorectal excision (TME) for rectal cancer. A total of 924 patients with rectal cancer underwent a low anterior resection. In 523 of those 924 patients (57\%), a primary stoma was created after a low anterior resection: 329 of those 523 patients (63\%) had ileostomies and 194 (37\%) had colostomies. The median follow-up was 7.1 years (range, 2.59.8 years). Of all stomas, $19 \%$ were never reversed during followup. The study concluded that a permanent stoma was highly related to postoperative complications (anastomotic leakage, abscess, sepsis, peritonitis, fistula, bleeding, stenosis, and others) and secondary constructed stomas.

When patients with rectal cancer successfully complete ileostomy closure, one would think that they would be content. However, another obstacle awaits them: fecal incontinence is a serious complication after an ileostomy reversal. Walma et al. [3] reported predictors of fecal incontinence and quality of life after a TME. For outcome measures, they used the fecal incontinence quality of life (FIQoL) scale [4] and the Wexner incontinence score [5]. They concluded that a diverting ileostomy is a predictor for an impaired FIQoL subscale coping/behavior after a TME. Ileostomy closure within 3 months presented a better functional outcome (Wexner scores, FIQoL total and all subscales) compared with ileostomy reversal after 3 months. They supposed that the etiology of this result might be a reduced functional adaptability of the neorectum after a long diversion. This result is very useful to surgeons who want to determine when to perform the ileostomy closure. However, the study had some limitations that need to be considered: First, the number of patients who underwent ileostomy closure within 3 months was small $(n=17)$ compared with the number of patients who underwent ileostomy closure after 3 months $(n=41)$. Second, although 
no statistically significant differences in the incidences of low rectal tumors $(\leq 5 \mathrm{~cm})$ were noted, the two groups in the study seemed to be quite different: 1 of 17 patients (5.9\%) underwent ileostomy closure within 3 months while 8 of 41 patients (19.5\%) underwent ileostomy closure after 3 months. As to the better functional outcomes reported in patients who underwent ileostomy closure within 3 months, the reader should cautiously consider the small number of patients and the low incidence of low rectal cancer. Can the timing of the ileostomy reversal influence functional outcome?

Conclusively, although some of the results reported in this paper may be subject to debate, the results do teach some important lessons; i.e., the ileostomy itself may be related to impaired FIQoL after ileostomy closure, and when no reason exists for a delay, ileostomy reversal within 3 months after primary surgery is recommended and is closely associated with better functional outcome.

\section{CONFLICT OF INTEREST}

No potential conflict of interest relevant to this article was reported.

\section{REFERENCES}

1. Chand M, Nash GF, Talbot RW. Timely closure of loop ileostomy following anterior resection for rectal cancer. Eur J Cancer Care 2008;17:611-5.

2. den Dulk M, Smit M, Peeters KC, Kranenbarg EM, Rutten HJ, Wiggers T, et al. A multivariate analysis of limiting factors for stoma reversal in patients with rectal cancer entered into the total mesorectal excision (TME) trial: a retrospective study. Lancet Oncol 2007;8:297-303.

3. Walma MS, Kornmann VN, Boerma D, de Roos MA, van Westreenen HL. Predictors of fecal incontinence and related quality of life after a total mesorectal excision with primary anastomosis for patients with rectal cancer. Ann Coloproctol 2015;31:23-8.

4. Rockwood TH, Church JM, Fleshman JW, Kane RL, Mavrantonis C, Thorson AG, et al. Fecal incontinence quality of life scale: quality of life instrument for patients with fecal incontinence. Dis Colon Rectum 2000;43:9-16.

5. Rockwood TH, Church JM, Fleshman JW, Kane RL, Mavrantonis C, Thorson AG, et al. Patient and surgeon ranking of the severity of symptoms associated with fecal incontinence: the fecal incontinence severity index. Dis Colon Rectum 1999;42:1525-32. 